\title{
Co-delivery and controlled release of stromal cell-derived factor-1 $\alpha$ chemically conjugated on collagen scaffolds enhances bone morphogenetic protein-2-driven osteogenesis in rats
}

\author{
HAIPENG SUN $^{1 *}$, JINMING WANG $^{1 *}$, FEILONG DENG ${ }^{1}$, YUN LIU $^{1}$, \\ XIUMEI ZHUANG ${ }^{2}$, JIAYUN XU' ${ }^{1}$ and LONG $\mathrm{LI}^{3}$
}

\begin{abstract}
${ }^{1}$ Department of Oral Implantology, Hospital of Stomatology, Guanghua School of Stomatology, Guangdong Provincial Key Laboratory of Stomatology; ${ }^{2}$ Department of Oral Implantology, Sun Yat-Sen Memorial Hospital;

${ }^{3}$ Department of Oral and Maxillofacial Surgery, Hospital of Stomatology, Guanghua School of Stomatology, Guangdong Provincial Key Laboratory of Stomatology, Sun Yat-Sen University, Guangzhou, Guangdong 510055, P.R. China
\end{abstract}

Received June 1, 2015; Accepted April 12, 2016

DOI: $10.3892 / \mathrm{mmr} .2016 .5339$

\begin{abstract}
There has been considerable focus in investigations on the delivery systems and clinical applications of bone morphogenetic protein-2 (BMP-2) for novel bone formation. However, current delivery systems require high levels of BMP-2 to exert a biological function. There are several concerns in using of high levels of BMP-2, including safety and the high cost of treatment. Therefore, the development of strategies to decrease the levels of BMP-2 required in these delivery systems is required. In our previous studies, a controlled-release system was developed, which used Traut's reagent and the cross-linker, 4-(N-maleimi-domethyl) cyclohexane-1-carboxylic acid 3-sulfo-N-hydroxysuccinimide ester sodium salt (Sulfo-SMCC), to chemically conjugate BMP-2 directly on collagen discs. In the current study, retention efficiency and release kinetics of stromal cell-derived factor- $1 \alpha$ (SDF- $1 \alpha$ ) cross-linked on collagen scaffolds were detected. In addition, the osteogenic activity of SDF-1 $\alpha$ and suboptimal doses of BMP-2 cross-linked on collagen discs following subcutaneous implantation in rats were evaluated. Independent two-tailed t-tests and one-way analysis of variance were used for analysis. In the present study, the controlled release of SDF-1 $\alpha$ chemically conjugated on collagen scaffolds was demonstrated. By optimizing the concentrations of
\end{abstract}

Correspondence to: Professor Feilong Deng, Department of Oral Implantology, Hospital of Stomatology, Guanghua School of Stomatology, Guangdong Provincial Key Laboratory of Stomatology, Sun Yat-Sen University, 56 Lingyuan Xi Road, Guangzhou, Guangdong 510055, P.R. China

E-mail: drdf1@163.com

*Contributed equally

Key words: collagen, chemically conjugated, co-delivery, stromal cell-derived factor-1 $\alpha$, bone morphogenetic protein-2, bone formation, controlled release
Traut's reagent and the Sulfo-SMCC cross-linker, a significantly higher level of SDF-1 $\alpha$ was covalently retained on the collagen scaffold, compared with that retained using a physical adsorption method. Mesenchymal stem cell homing indicated that the biological function of the SDF-1 $\alpha$ cross-linked on the collagen scaffolds remained intact. In rats, co-treatment with SDF- $1 \alpha$ and a suboptimal dose of BMP-2 cross-linked on collagen scaffolds using this chemically conjugated method induced higher levels of ectopic bone formation, compared with the physical adsorption method. No ectopic bone formation was observed following treatment with a suboptimal dose of BMP-2 alone. Therefore, the co-delivery of SDF-1 $\alpha$ and a suboptimal dose of BMP-2 chemically conjugated on collagen scaffolds for the treatment of bone injuries reduced the level of BMP-2 required, reducing the risks of side effects.

\section{Introduction}

Bone graft procedures are frequently performed in oral and maxillofacial surgery to treat defects of the maxilla or mandible resulting from tumor resection, fractures, infection and skeletal dysplasia (1). Currently, the gold standard approach involves autograft bone treatment, however, autograft bone is not readily available and is associated with donor site morbidity $(2,3)$. Allograft bone and xenograft bone are feasible alternatives, however, the risks of host rejection, disease transmission and infections have limited their use (4).

Bone formation is a complex process, which involves interactions between different cells, growth factors and extracellular matrix to promote the proliferation, differentiation and migration of osteoprogenitor cells $(5,6)$. The application of growth factors to induce the natural healing of bone and promote bone tissue remodeling may be a possible strategy to improve current approaches for bone regeneration. During laboratory experiments and clinical trials, growth factors have exhibited promising therapeutic potential, however, few have been approved for clinical use (4). Bone morphogenetic protein-2 (BMP-2) has been approved for clinical application since 2002 (1). BMP-2 has been successfully used in non-union fractures, joint fusions, critical 
bone defects and aseptic bone necrosis, which demonstrated its use as a possible alternative to autografts (7-9). However, soluble BMP-2 is rapidly degraded by proteolytic enzymes in the body, making it difficult to achieve a stable maintenance dose for treatment throughout the period of bone regeneration. Furthermore, particularly in larger animal models and humans, current delivery methods require high levels of BMP-2 to exert a biological function (10). Using of high levels of BMP-2 is associated with several issues, including safety and the high cost of treatment (11). Several strategies are being developed to decrease the high doses of BMP-2 required, one of which is to develop a controlled-release delivery system, which not only prevents the fast degradation of BMP-2, but also provides continued release throughout the entire process of bone formation (12-14). Other possible approaches include augmenting bone healing using different growth factors, cytokines or chemokines as co-therapeutics with treatments such as BMP-2 (15-17).

Stromal cell-derived factor-1 (SDF-1) belongs to the proinflammatory CXC chemokine family (18); its receptor is CXC chemokine receptor 4 (CXCR4) and they are expressed in various tissues $(19,20)$. The binding of SDF-1 to its complementary receptor, CXCR4, induces the homing of stem cells to the bone marrow, and previous investigations have demonstrated that SDF-1 is an important chemokine in recruiting stem and progenitor cells for tissue restoration following injury, including during the acute phase of bone repair (21-24). Several reports have also shown that SDF-1 directly regulates the signaling during BMP-2-induced mesenchymal cell progression towards osteogenic differentiation in vitro $(25,26)$ and in vivo $(27-29)$. In our previous study, a controlled-release system of BMP-2 cross-linked on a collagen disc was developed. The BMP-2 was chemically conjugated onto the collagen disc using Traut's reagent and the cross-linker, 4-(N-maleimi-domethyl) cyclohexane-1-carboxylic acid 3-sulfo-N-hydroxysuccinimide ester sodium salt (Sulfo-SMCC) (30). This delivery system was found to be suitable for use in bone tissue engineering.

In the present study, the chemical combination method using Traut's reagent and the Sulfo-SMCC cross-linker was adapted to chemically conjugate SDF-1 $\alpha$ on collagen discs. It was hypothesized that this method can significantly increase the retention of SDF-1 $\alpha$ on the collagen scaffold without decreasing its biological activity, and reduce the rate of release. The SDF- $1 \alpha$ binding and release rates were determined following cross-linking in vitro, and cell homing assays were performed to investigate the biological function of SDF- $1 \alpha$ chemically conjugated on the collagen disc. It was further hypothesized that the co-delivery of BMP-2 and SDF-1 $\alpha$ cross-linked on collagen scaffolds enhances bone formation, whilst requiring significantly reduced levels of BMP-2. By evaluating ectopic bone growth 28 days following implantation in rats, the present study investigated the cooperative osteogenic effect of SDF-1 $\alpha$ and a suboptimal dose of BMP-2, which were cross-linked on separate collagen discs. The current study aimed to investigate whether the strategy investigated may enable reductions in BMP-2 doses, consequently reducing the risk of side effects and the cost of therapy.

\section{Materials and methods}

Collagen discs. The collagen membranes, derived from ox leather, (Fig. 1A) were provided by Zhenghai Biotechnology
(Shandong, China). The uniform round collagen discs $(6.4 \mathrm{~mm}$ diameter; $0.5 \mathrm{~mm}$ thickness; Fig. 1B) were sterilized using 12 kGy Co-60 irradiation (GM-II; Beijing Gamma High-tech Co., Ltd., Beijing, China) and were prepared, cut with a hole puncher into uniform discs with a diameter of $6.4 \mathrm{~mm}$ and thickness of $0.5 \mathrm{~mm}$, as described previously $(30,31)$. The collagen discs were observed under a scanning electron microscope (SEM; model S-4800; Hitachi, Tokyo, Japan; Fig. 1C).

Collagen scaffold modification using Traut's reagent. Sulfhydryl (SH) groups were added to the collagen discs, according to previously described methods (31). Briefly, Traut's reagent (2-iminothiolane hydrochloride; Thermo Fisher Scientific, Inc., Rockford, IL, USA) was dissolved in phosphate-buffered saline (PBS) and $4 \mathrm{mM}$ ethylenediamine tetraacetic acid disodium (EDTA; Guangzhou Chemical Reagent Company, Guangzhou, China), with a final concentration of $2.5 \mathrm{mg} \mathrm{ml}^{-1}$. The collagen discs were placed into $100 \mu \mathrm{l}$ of Traut's reagent solution in a 96-well plate. After $12 \mathrm{~h}$ incubation at $4^{\circ} \mathrm{C}$, the collagen-SH samples were rinsed three times with $100 \mu \mathrm{l}$ PBS and $4 \mathrm{mM}$ EDTA (5 min/wash).

Cross-linking of SDF-1 $\alpha$ on the collagen disc using Sulfo-SMCC. According to the manufacturer's protocol, a 50-fold molar overdose of Sulfo-SMCC (Sigma-Aldrich, St. Louis, MO, USA) was mixed with SDF-1 $\alpha$ (recombinant rat SDF-1 $\alpha$; PeproTech, Rocky Hill, NJ, USA) at room temperature for $5 \mathrm{~min}$. The SDF-1-SMCC complex solution $(100 \mu \mathrm{l})$ was then incubated with the collagen-SH for $1 \mathrm{~h}$ at $4^{\circ} \mathrm{C}$ to form the collagen-SH-SMCC construct. The collagen-SH-SMCC construct was rinsed three times with PBS (5 min/wash) on a shaker to remove any non-binding SDF-1 $\alpha$ or excess cross-linking agent.

Efficiency of SDF-1a retention on the collagen scaffolds. The collagen discs were divided randomly into a cross-linked collagen group and a physical adsorbed collagen group to assess the efficiency of the conjugation of SDF-1 $\alpha$. The collagen discs in the cross-linked group were modified using Traut's reagent, as described above, and serially diluted SDF-1 $\alpha(20,10,5,2.5,1.25$ and $0 \mu \mathrm{gml}^{-1}$ ) was combined with the Sulfo-SMCC, as described above. For each SDF-1 $\alpha$ concentration, the collagen-SH was soaked in $100 \mu \mathrm{l}$ of the SDF-1 $\alpha$-SMCC solution for $1 \mathrm{~h}$ at $4^{\circ} \mathrm{C}$. The collagen discs in the physical adsorbed collagen group were soaked in $100 \mu \mathrm{lPBS}$ for $12 \mathrm{~h}$ at $4^{\circ} \mathrm{C}$, and were then immersed in each SDF-1 $\alpha$ concentration solution in PBS for $1 \mathrm{~h}$ at $4^{\circ} \mathrm{C}$. The cross-linked collagen group and the physical adsorbed collagen group were then washed with PBS (three times; $5 \mathrm{~min} /$ wash) to remove any non-binding SDF-1 $\alpha$ or excess cross-linking agent.

The present study used the improved direct enzyme-linked immunosorbent assay (ELISA) technique to quantify the retention of SDF-1 $\alpha$ on the collagen discs; this improved ELISA was successfully used in our previous studies and those of others (30-33). Briefly, the discs were blocked with $100 \mu$ l bovine serum albumin (50 $\mathrm{mg} \mathrm{ml}^{-1}$; MP Biomedicals, Solon, OH, USA) in PBS for $1 \mathrm{~h}$ at room temperature in a 96-well plate. Following washing twice in PBS with $0.05 \%$ Tween-20 (PBST), the discs were incubated with $100 \mu 1$ rabbit polyclonal antibody to SDF-1 $\alpha$ (1:1,000 in PBS; ab9797; Abcam, Cambridge UK) for $1 \mathrm{~h}$ at room temperature. Following washing three times in PBST for $5 \mathrm{~min}, 100 \mu \mathrm{l}$ of the AffiniPure goat anti-rabbit $\operatorname{IgG}(1: 10,000$ in 
PBS; E030220-01; EarthOx Life Sciences, San Francisco, CA, USA) conjugated with alkaline phosphatase (AP) was added for $1 \mathrm{~h}$ at room temperature. Following washing, as above, three times, $200 \mu \mathrm{l}$ of $2 \mathrm{mg} \mathrm{ml}^{-1}$ phosphorylated-nitrophenyl phosphate hexahydrate (Sigma-Aldrich) in AP buffer, containing $100 \mathrm{mM} \mathrm{NaCl}, 10 \mathrm{mM} \mathrm{MgCl}_{2}$ and $100 \mathrm{mM}$ Tris $\mathrm{HCl}(\mathrm{pH}$ 9.6) was added for $15 \mathrm{~min}$. The bound protein was determined by removing $100 \mu \mathrm{l}$ of the colored substrate solution into a separate 96-well plate and measuring at an absorbance/optical density (OD) of $405 \mathrm{~nm}$ (OD405) in an ELISA reader (Infinite 2000; Tecan Group, Ltd., Männedorf, Switzerland).

Release kinetics of SDF-1 $\alpha$ cross-linked on collagen scaffolds. SDF-1 $\alpha(1 \mu \mathrm{g}$ in $100 \mu \mathrm{l})$ was loaded onto collagen discs using the cross-linking or physical adsorption techniques, according to the methods described above. The collagen discs were immersed in $100 \mu 1 \mathrm{PBS}+0.02 \%$ sodium azide (Sigma-Aldrich). After $0,12,24,48,72,96$ and $120 \mathrm{~h}$ on a shaker at $37^{\circ} \mathrm{C}$, the dose of SDF-1 $\alpha$ immobilized on the collagen discs at each time point was analyzed using ELISA, as described above.

In vitro cell-homing. The bioactivity of SDF-1 $\alpha$ cross-linked on collagen was assessed by analyzing the chemotaxis of mesenchymal stem cells (MSCs) in response to the SDF-1 $\alpha$ stimulus using a Transwell (Boyden chamber) migration assay. Sprague-Dawley rat bone MSCs were isolated and purified for culture in vitro and serial passage by the attachment culture method in basal cell culture media. The third-generation cells were digested with $0.25 \%$ trypsin and $0.02 \%$ EDTA to obtain a single-cell suspension. The rat MSCs $\left(2 \times 10^{4}\right.$ cells in $250 \mu \mathrm{l}$ ) were placed in the upper chamber of 24-well Transwell inserts containing polycarbonate membranes with $8-\mu \mathrm{m}$ pores (Corning Costar, Corning, NY, USA). Blank collagen scaffolds and the collagen scaffolds cross-linked or physically adsorbed with SDF-1 $\alpha(1 \mu \mathrm{g})$ were placed in the lower chamber of each Transwell containing $500 \mu 1$ of Dulbecco's modified Eagle's medium (Thermo Fisher Scientific, Inc.) supplemented with $2 \%$ fetal bovin serum (Thermo Fisher Scientific, Inc.). Following incubation at $37^{\circ} \mathrm{C}$ for $24 \mathrm{~h}$, the cells were fixed in $4 \%$ paraformaldehyde (Guangzhou Chemical Reagent Company) for $15 \mathrm{~min}$. The cells retained on top of the polycarbonate membrane of each Transwell were removed. The migrated cells in the lower chamber of the Transwells were analyzed by staining with 4',6-diamidino-2-phenylindole (DAPI; Beijing Leagene Biotechnology Co., Ltd., Beijing, China) and were detected in five randomly selected fields $\left(0.25 \mathrm{~mm}^{2}\right.$; magnification, $\mathrm{x} 200$; Axiovert $40 \mathrm{C}$ inverted fluorescence microscope; Zeiss, Oberkochen, Germany). The mean numbers of cells from the five fields were calculated. The experiments were repeated three times.

Osteogenic activity of SDF-1 $\alpha$ and suboptimal doses of BMP-2 cross-linked on collagen discs following subcutaneous implantation in rats. A total of 24 male Sprague-Dawley rats (weight, 220-250 g; age, 6 8 weeks) were housed in a temperature- $\left(24 \sim 26^{\circ} \mathrm{C}\right)$ and light- $(12 / 12 \mathrm{~h}$ light/dark cycle) controlled environment, with free access to food and water. The rats were used to evaluate the osteogenic activity of SDF-1 $\alpha$ and suboptimal doses of BMP- 2 cross-linked on collagen discs. The animal experiments were approved by the Animal Care and
Research Committee of Sun Yat-sen University (Guangzhou, China). Animal care and surgical procedures were performed in accordance with the guidelines of Guangdong Association on Laboratory Animal Care (Guangdong, China).

BMP-2 (2.5 $\mu$ g rhBMP-2; PeproTech, Rocky Hill, NJ, USA) and SDF-1 $\alpha$ (1 $\mu \mathrm{g}$ rmSDF-1 $\alpha$; PeproTech) were loaded onto separate sterile collagen discs in a biosafety cabinet and transported for surgery in sterile boxes. Surgery was performed with the animals above a heated pad. The rats were anesthetized via an intraperitoneal injection of $30 \mathrm{mg} \mathrm{kg}^{-1}$ pentobarbital sodium (Military Veterinary Institute, Changchun, China). The dorsal surface of the implant sites were shaved and disinfected using a 10\% betadine solution (Guangzhou Chemical Reagent Company). A total of four $0.8-\mathrm{cm}$ transverse incisions were made, $\sim 3 \mathrm{~cm}$ apart, on the dorsal midline of the rat, and the skin and subcutaneous tissues were bluntly dissected to form subcutaneous pockets. The four groups of implants were as follows: i) Blank control group, the implant contained two blank collagen scaffolds; ii) suboptimal BMP-2-cross-linked group, the implant contained one blank collagen scaffold and one collagen scaffold cross-linked with $2.5 \mu \mathrm{g}$ BMP-2; iii) SDF-1 $\alpha /$ suboptimal BMP-2-physically adsorbed collagen group, the implant contained one collagen scaffold adsorbed with $1 \mu \mathrm{g}$ SDF-1 $\alpha$ and one collagen scaffold adsorbed with $2.5 \mu \mathrm{g}$ BMP-2; iv) SDF-1 $\alpha$ /suboptimal BMP-2-cross-linked group, the implant contained one collagen scaffold cross-linked with $1 \mu \mathrm{g} \mathrm{SDF}-1 \alpha$ and one collagen scaffold cross-linked with $2.5 \mu \mathrm{g}$ BMP-2. The two overlapped discs were fixed with absorbable sutures (Vicryl; Johnson \& Johnson, Shanghai, China) forming a compound implant. The four groups of implants were randomly inserted into the pockets of each rat. The rats were housed with one animal/cage and fed a normal diet and water. A total of six animals were sacrificed 4 weeks post-implantation by injection with an overdose of pentobarbital sodium, and the discs were carefully removed. The specimens were fixed in $4 \%$ paraformaldehyde for 2 days, embedded in paraffin (Guangzhou Chemical Reagent Company) and cut into $5-\mu \mathrm{m}$ sections. The sections were stained with von Kossa staining ( $1 \%$ silver nitrate solution and counterstained with hematoxylin and eosin staining; Guangzhou Chemical Reagent Company). Quantitative analysis of the percentage of calcium content was performed using Image-Pro Plus 6.0 software (Media Cybernetics, Inc., Silver Spring, MD, USA).

Statistical analysis. All statistical data were analyzed using SPSS 16.0 software (SPSS, Inc., Chicago, IL, USA). Independent two-tailed $t$-tests were used for two group comparison, and one-way analysis of variance, followed by Tukey's test was used for multiple group comparisons. All data are expressed as the mean \pm standard deviation. $\mathrm{P}<0.05$ was considered to indicate a statistically significant difference.

\section{Results and Discussion}

Properties of collagen membranes. The SEM examination of the collagen membranes, obtained from Zhenghai Biotechnology, showed that the surface contained multiple pores (range, 50-200 $\mu \mathrm{m}$ ), and collagen fibers were loosely packed and linked with each other to form a three-dimensional network structure (Fig. 1C). This pore size range is conducive 

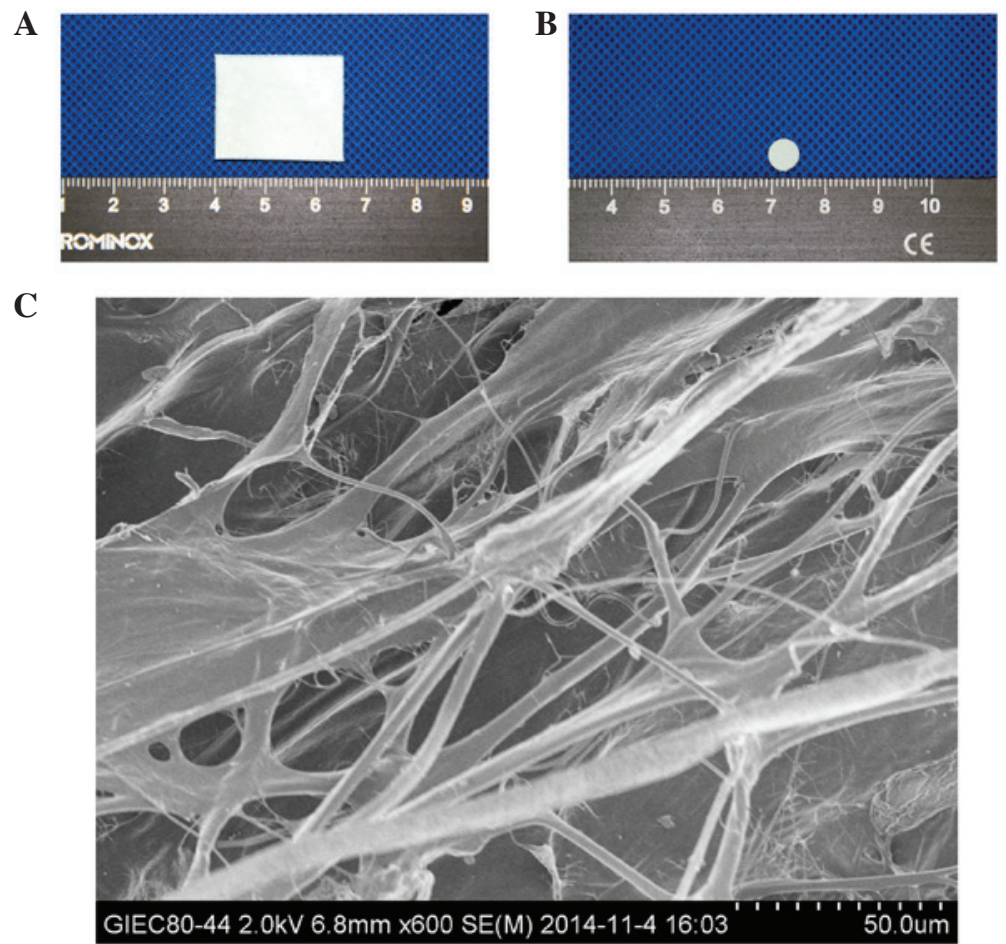

Figure 1. Collagen disc dimensions. (A) Overview of collagen scaffold (length, $2.5 \mathrm{~cm}$; width, $2 \mathrm{~cm}$ ). (B) Uniform round collagen disc (diameter, $6.4 \mathrm{~mm}$; thickness, $\mathrm{x} 0.5 \mathrm{~mm}$ ). (C) Scanning electron microscopy of the collagen disc (scale bar=50 $\mu \mathrm{m}$ ).

A<smiles>[SiH3]NC1CCCS1</smiles>

Traut's Reagent $\begin{aligned} & \begin{array}{l}\text { Collagen containing } \\ \text { primary amine }\end{array} \quad \text { Collagen-SH } \\ & \text { pring }\end{aligned}$

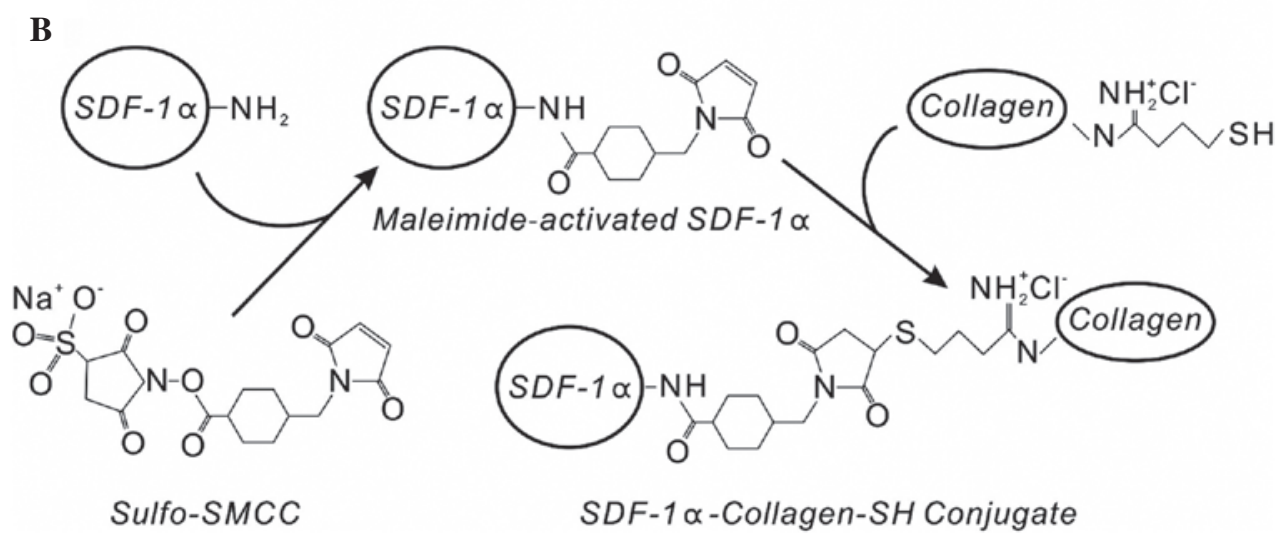

Figure 2. Collagen-SH formation and conjugation with SDF-1 $\alpha$. (A) Molecular structure of Traut's reagent and its interaction with the collagen scaffold. (B) Conjugation of SDF-1 $\alpha$ on the collagen-SH scaffold using Sulfo-SMCC. SDF-1 $\alpha$, stromal cell-derived factor-1 $\alpha$; Sulfo-SMCC, 4-(N-maleimi-domethyl) cyclohexane-1-carboxylic acid 3-sulfo-N-hydroxysuccinimide ester sodium salt.

to the attachment of cells and growth factors (31), and the stable and mechanical structure of the collagen were suitable for cell guidance and attachment. Collagen has several beneficial properties, including low antigenicity, biocompatibility and biodegradability $(34,35)$, which has led to it being widely used in the development and wound repair of organs and tissues, including skin, bone and the vascular system (36-38).
SDF-1 $\alpha$ conjugation on collagen-SH discs using Sulfo-SMCC. In our previous experiment, collagen-SH discs (Fig. 2A) were formed by treating collagen membranes with Traut's reagent, which can add $\mathrm{SH}$ groups to primary amines (39). It was found that saturation of the $\mathrm{SH}$ groups on the small round collagen discs was achieved at a concentration of $2.5 \mathrm{mg} \mathrm{ml}^{-1}$ of Traut's reagent (31). The cross-linker, Sulfo-SMCC, was then used 


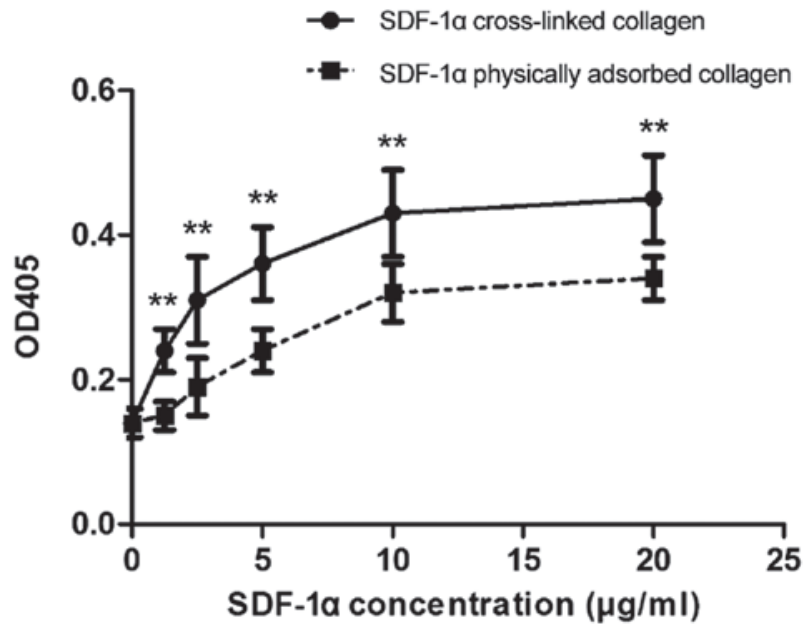

Figure 3. Binding curves for SDF-1 $\alpha$ with the Sulfo-SMCC cross-linker and collagen-SH (SDF-1 $\alpha$ cross-linked collagen group), and with phosphate-buffered saline and collagen (SDF-1 $\alpha$ physically adsorbed collagen group). ELISA was used to obtain measurements. Data are expressed as the mean \pm standard deviation, ${ }^{* *} \mathrm{P}<0.01$. SDF-1 $\alpha$, stromal cell-derived factor- $1 \alpha$; Sulfo-SMCC, 4-(N-maleimi-domethyl) cyclohexane-1-carboxylic acid 3-sulfo-N-hydroxysuccinimide ester sodium salt; OD, optical density.

to conjugate vascular endothelial growth factor (VEGF) or BMP-2 on the collagen-SH scaffolds, and the biological function of these growth factors was maintained $(30,31)$.

In the present study, SDF-1 $\alpha$ was cross-linked on the collagen scaffolds using the above strategy with Traut's reagent and Sulfo-SMCC. The same $\left(2.5 \mathrm{mg} \mathrm{ml}^{-1}\right)$ concentration of Traut's reagent was used to form the collagen-SH scaffolds. The Sulfo-SMCC cross-linker reagent can covalently conjugate amine- and SH-containing molecules. The amino groups of SDF- $1 \alpha$ can create amide bonds with the maleimide groups of Sulfo-SMCC to form maleimide-activated SDF-1 $\alpha$ (Fig. 2B), which selectively reacts with the collagen-SH scaffolds made by Traut's reagent. The results from the ELISA indicated that SDF- $1 \alpha$ at concentrations $>1.25 \mu \mathrm{g} \mathrm{ml}^{-1}$ showed improved immobilization on the collagen-SH disc using Sulfo-SMCC, compared with physical adsorption (Fig. 3). The immobilized SDF-1 $\alpha$ directly bound to the collagen scaffold reduced its distribution and degradation, thus reducing the concentration of SDF-1 $\alpha$ required. The covalently conjugated SDF-1 $\alpha$ on the collagen scaffold was able to maintain an effective concentration of SDF-1 $\alpha$ at the target location.

Release kinetics of SDF-1 $\alpha$ post cross-linkage. The release of SDF-1 $\alpha$ cross-linked or physically adsorbed on the collagen scaffolds within a $120 \mathrm{~h}$ period was detected by analyzing the remaining protein on the collagen scaffolds at each time point (Fig. 4). The cross-linked SDF-1 $\alpha$ showed decreased release, compared with the physically adsorbed SDF-1 $\alpha$, and this release occurred in a controlled time-dependent manner within the $120 \mathrm{~h}$ period. The physically adsorbed SDF- $1 \alpha$ typically exhibited rapid release during the first $24 \mathrm{~h}$, releasing almost $60 \%$ of the SDF- $1 \alpha$ adsorbed on the collagen scaffold, compared with $20 \%$ of the SDF-1 $\alpha$ cross-linked on the collagen scaffold in the same period. Although equal doses of SDF-1 $\alpha$ were applied on the collagen scaffolds in each group, a high level of SDF-1 $\alpha$ remained in the cross-linked group,

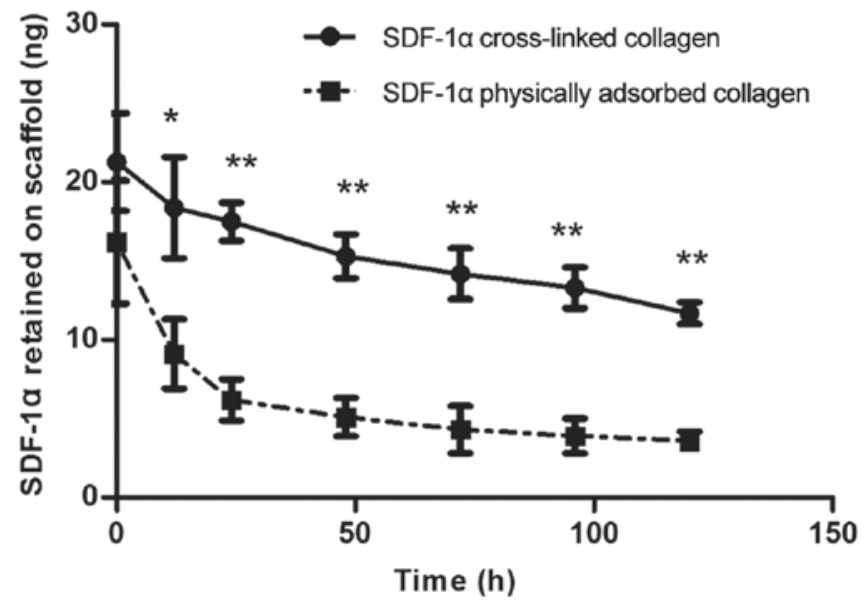

Figure 4. Release kinetics of SDF-1 $\alpha$ from the collagen scaffold in vitro. Data are expressed as the mean \pm standard deviation, ${ }^{*} \mathrm{P}<0.05,{ }^{* *} \mathrm{P}<0.01$. SDF- $1 \alpha$, stromal cell-derived factor- $1 \alpha$.

compared with the physical adsorption group at $0 \mathrm{~h}$, even with repeated washes during the assessment, which may be attributed to the manner of the binding of SDF-1 $\alpha$. These results verified that cross-linked collagen scaffolds may be used for sustained chemokine-release.

Therapeutic growth factors are required to bind non-specifically to tissue constructs, maintain their conformation, and be released in a manner that does not affect their biological functions. Optimal carriers of therapeutic growth factors have two predominant properties: i) The degradation rate of the carrier is consistent with the rate of tissue repair from several weeks to several months, with maintained release of appropriate growth factors; and ii) have a high surface area to volume ratio and a sufficient number of interconnected pores to provide sufficient space, and a conducive environment for cellular growth and neovascularization (4). Collagen, the predominant protein component of natural extracellular matrix, has been extensively investigated as a natural scaffold in tissue engineering, and its capability to deliver growth factors to induce bone formation has been well established (40). In our previous study, it was confirmed that the degradation rate of cross-linked collagen discs was reduced, compared with normal collagen discs, which was of benefit to tissue restoration (31). In the present study, the ability to control the slow release of SDF-1 $\alpha$ conjugated to collagen improved its effectiveness as a cell-homing agent by reducing the requirement for continuous replenishment of the SDF-1 $\alpha$, which is constantly lost by diffusion and/or degradation by exopeptidases and matrix metalloproteinases in an inflammatory environment in vivo (41).

In vitro cell-homing. The bioactivity of SDF-1 $\alpha$ cross-linked on collagen scaffolds was evaluated using a modified Boyden chamber (Transwell migration) assay, to determine whether the released SDF-1 $\alpha$ maintained its biological function as a chemoattractant for stem cells. As shown in Fig. 5A and B, the nuclei of the migrated MSCs were stained blue with DAPI, and increased MSC migration was observed from the top to the bottom of the Transwell in the SDF-1 $\alpha$ cross-linked collagen group, compared with the blank collagen group. The chemotactic effect of the SDF-1 $\alpha$ released from chemokine-loaded 
A

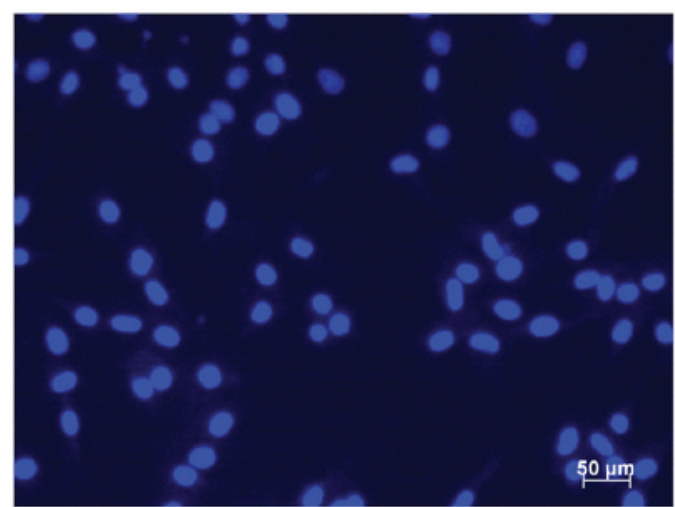

B

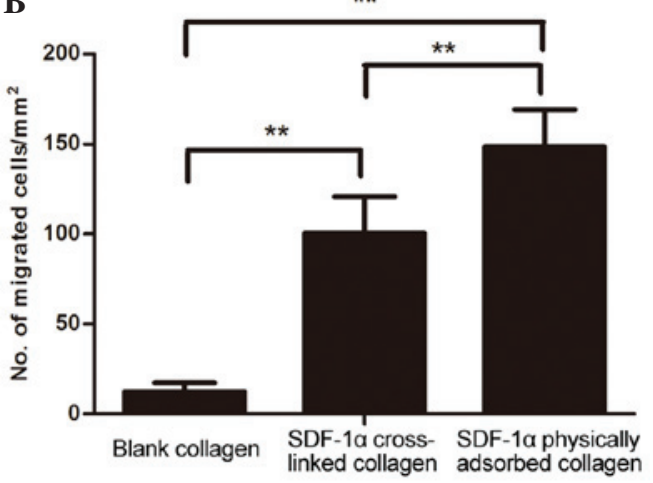

Figure 5. Ability of SDF-1 $\alpha$-loaded collagen scaffolds to act as a chemoattractant for mesenchymal stem cells. (A) 4',6-diamidino-2-phenylindole-stained image of the migrated mesenchymal stem cells in the lower Transwell chamber; scale bar, $50 \mu \mathrm{m}$. (B) Results of cell homing in the blank collagen, SDF-1 $\alpha$ cross-linked collagen and SDF- $1 \alpha$ physically adsorbed collagen groups. Data are expressed as the mean \pm standard deviation, ${ }^{* *} \mathrm{P}<0.01$. SDF-1 $\alpha$, stromal cell-derived factor- $1 \alpha$.

A

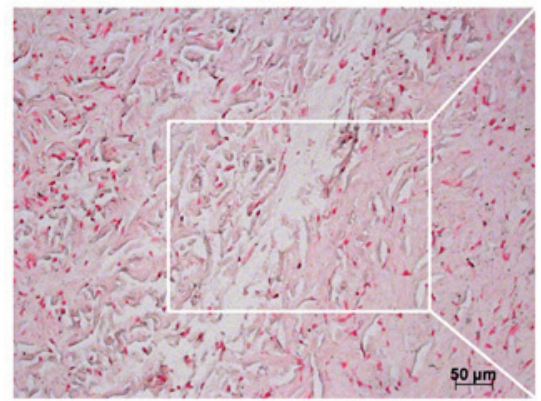

C

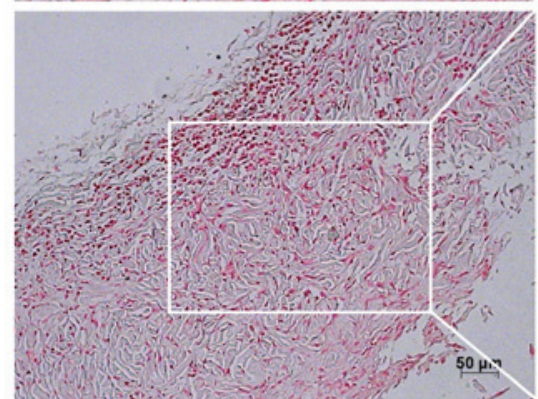

E

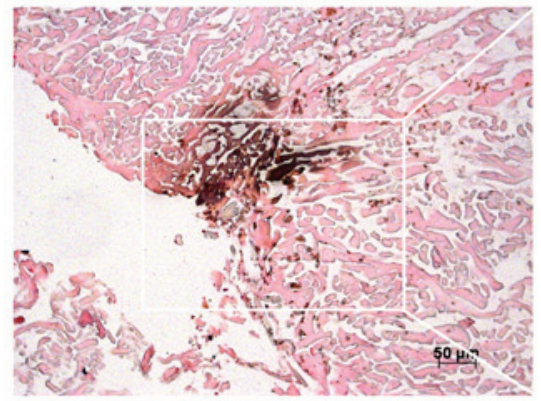

G

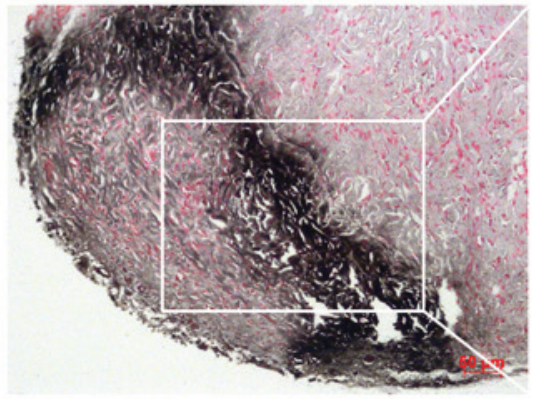

B

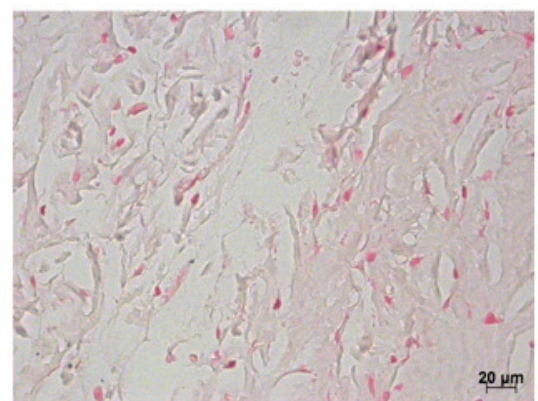

D

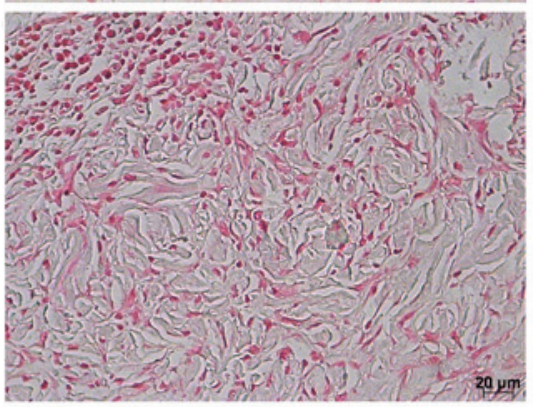

F

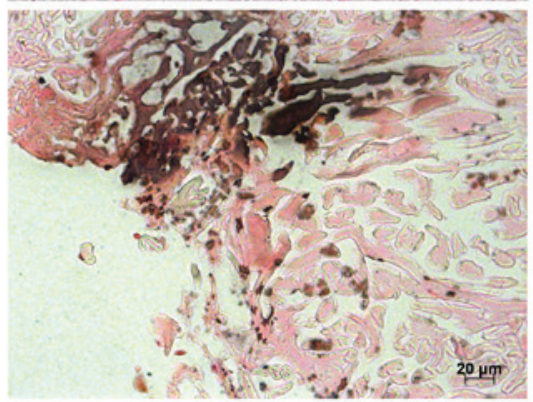

H

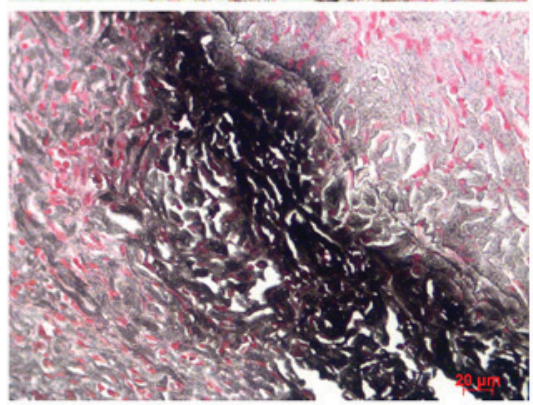

Figure 6. Von Kossa staining 4 weeks post-implantation. The black/brown staining shows positive calcium deposition. (A and B) The blank collagen group; (C and D) the low dose BMP-2 cross-linked collagen group; (E and F) the SDF-1 $\alpha$ /low dose BMP-2-physically adsorbed collagen group; and (G and H) the SDF-1 $\alpha$ /low dose BMP-2 cross-linked collagen group. The white frames indicate the magnification areas. $(\mathrm{A}, \mathrm{C}, \mathrm{E}, \mathrm{G}), \mathrm{scale}$ bar $=50 \mu \mathrm{m}, \mathrm{magnification}, \mathrm{x} 200$; $(\mathrm{B}, \mathrm{D}, \mathrm{F}, \mathrm{H})$, scale bar $=20 \mu \mathrm{m}$, magnification, $\mathrm{x} 400$. SDF-1 $\alpha$, stromal cell-derived factor-1 $\alpha$; BMP-2, bone morphogenetic protein-2. 
collagen scaffolds caused the MSCs to migrate from the top to the bottom of the Transwells. Compared with the SDF-1 $\alpha$ physically absorbed collagen group, the SDF-1 $\alpha$ cross-linked collagen group exhibited significantly reduced homing effects, which may be explained by the burst release of SDF-1 $\alpha$ in the SDF-1 $\alpha$ physical absorbed collagen group during the first $24 \mathrm{~h}$. It was concluded that SDF-1 $\alpha$ retained on cross-linking collagen scaffolds had preserved biological activity and slower release.

Ectopic bone formation in vivo. In addition to strategies, which reduce the high levels of BMP-2 required, other approaches incorporate the co-delivery of different growth factors, cytokines or chemokines with treatments, including BMP-2, to augment bone healing. These approaches simultaneously deliver two growth factors to a target site. However, each growth factor possesses different functions to regenerate tissues, including bone and blood vessels, requiring appropriate combinations of growth factors for cooperative action in regulating intact tissue regeneration (4).

In the present study, a controlled-release system for SDF-1 $\alpha$ and BMP-2 cross-linked on collagen scaffolds was developed using the chemical conjugate method with Traut's reagent and the Sulfo-SMCC cross-linker. The SDF-1 $\alpha$ cross-linked on the collagen disc almost reached saturation at concentrations $>10 \mu \mathrm{g} \mathrm{ml}^{-1}$ SDF-1 $\alpha$. For the BMP-2 cross-linked on the collagen scaffold, the same conditions were used as in our previous study, and were those of Higashino et al with modification (28), in which a minimum threshold dose of rhBMP-2 $(2.5 \mu \mathrm{g})$ as the suboptimal dose on each collagen pellet was used in an athymic rat ectopic bone formation model. A BMP-2 concentration of $25 \mu \mathrm{g} \mathrm{ml}^{-1}(2.5 \mu \mathrm{g}$ in $100 \mu \mathrm{l})$ was used as the suboptimal dose for cross-linking on the collagen scaffold. Each implant site contained two collagen membranes fixed by absorbable sutures, and each collagen membrane delivered one growth factor conjugated on the collagen scaffold. This method was simple and feasible as a delivery mechanism.

The present study analyzed the ectopic osteogenic effects of the blank collagen group, sub-optimal BMP-2-cross-linked collagen group, SDF-1 $\alpha /$ suboptimal BMP-2-physically adsorbed collagen group and SDF-1 $\alpha /$ suboptimal BMP-2-cross-linked collagen group using von Kossa staining. The von Kossa staining technique can detect deposits of calcium or calcium salt, with positive staining shown as dark brown regions, indicating the presence of mineral content (42). The newly formed bone was quantified by analyzing the percentage of calcified bone tissue. Negative staining in the blank group and suboptimal BMP-2-cross-linked group were observed. The SDF-1 $\alpha /$ suboptimal BMP-2-physically adsorbed group and SDF-1 $\alpha$ /suboptimal BMP-2-cross-linked group exhibited dark brown staining, indicating bone formation (Fig. 6). The quantification of the percentages of calcified tissue showed that the SDF-1 $\alpha /$ suboptimal BMP-2-cross-linked group $(31.86 \pm 7.10 \%)$ induced a high level of calcium deposition, compared with the SDF-1 $\alpha$ /suboptimal BMP-2-physically adsorbed group (9.82 $\pm 1.39 \%$; Fig. 7). Statistical analysis revealed that the difference between the two groups was significant $(\mathrm{P}<0.01)$. Furthermore, SDF-1 $\alpha /$ suboptimal BMP-2 cross-linked group showed higher calcium density, as indicated by darker positive staining, compared with the SDF-1 $\alpha$ /subop-

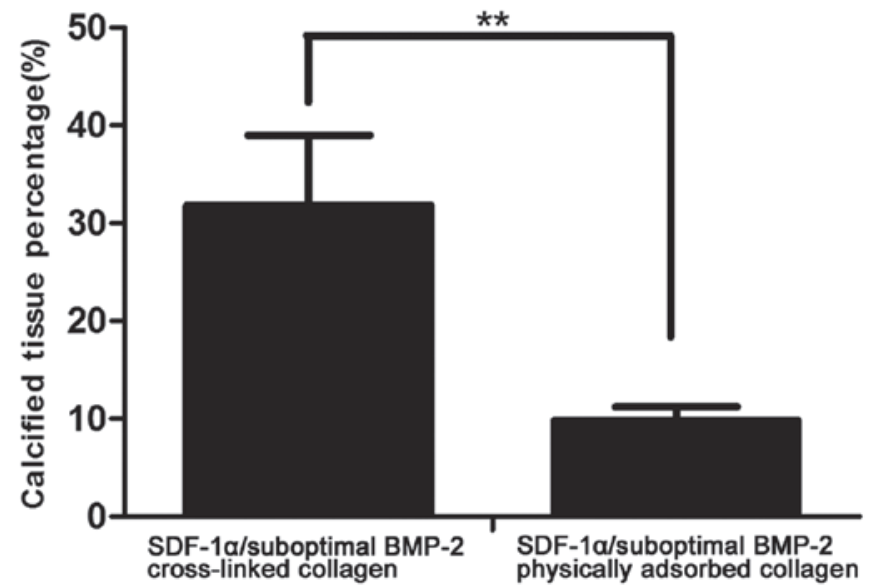

Figure 7. Percentages of calcified tissue in the SDF-1 $\alpha /$ suboptimal BMP-2 cross-linked collagen group and SDF-1 $\alpha$ /suboptimal BMP-2 physically adsorbed collagen group. Data are expressed as the mean \pm standard deviation, ${ }^{* *} \mathrm{P}<0.01$. SDF-1 $\alpha$, stromal cell-derived factor-1 $\alpha$; BMP-2, bone morphogenetic protein-2.

timal BMP-2-physically adsorbed group. This indicated that the rates in the SDF-1 $\alpha$ /suboptimal BMP-2-cross-linked group had a higher level of ectopic bone regeneration. These findings further verified that this delivery vehicle was effective for growth factors, and they showed that SDF- $1 \alpha$ potentiated suboptimal levels of BMP-2 to induce bone regeneration, whereas suboptimal BMP-2 alone was not able to induce bone regeneration.

Richardson et al reported on a novel vehicle, which allowed for the tissue-specific delivery of two or more growth factors with distinct kinetics, and their findings showed that the dual delivery of VEGF-165 and platelet-derived growth factor-BB resulted in the rapid formation of a mature vascular network (43). The cooperative effects of the co-delivery of BMP-2 with growth factors, including VEGF, transforming growth factor $\beta$-3 or insulin-like growth factor-1 have been investigated in vitro and in vivo using structural polymer scaffolds for bone tissue regeneration and engineering utility (44-47). Previously, several studies have focused on the cooperative effects of BMP-2 and SDF-1. Herberg et al reported that SDF-1 $\beta$ had potent synergistic effects on BMP-induced local bone formation and may be a suitable candidate for the optimization of bone augmentation, which used significantly lower levels of BMP-2 for spinal, orthopedic and craniofacial treatments (27). In this previous study, BMP-2 and SDF-1 were physically adsorbed onto a collagen sponge by soak-loading. Higashino et al reported that the addition of SDF-1 to implants containing a suboptimal level of BMP-2 enhanced the mobilization and migration of MOPCs to the implant and induced ectopic bone formation (28). BMP-2 and SDF-1 were also physically adsorbed on the collagen pellets by soak-loading. There are certain limitations, including higher levels of growth factors and rapid release, in the reports on the physical adsorption of growth factors by soak-loading. Herberg et al also reported on a delivery system for the sustained release of a low-dose growth factor using biopatterning technology, which assisted in the healing of CSD injuries. It was found that SDF-1 $\beta$ augmented the ability of BMP-2 to drive the healing (48). The 
biopatterning technology was used to achieve the controlled release of growth factors. In the present study, SDF-1 $\alpha$ and BMP-2 directly cross-linked on collagen were used to obtain slow controlled release and reduce the dose of growth factors required. The results of the present study conceptualize a process whereby the initial release of SDF- $1 \alpha$ from a collagen scaffold may stimulate stem cell migration to the collagen scaffold, which subsequently promoted bone formation in the presence of suboptimal levels of BMP-2.

The findings from the present study indicated that a higher level of SDF-1 $\alpha$ was retained on the collagen disc applying Traut's reagent and the Sulfo-SMCC cross-linker, with intact biological function. Accordingly, the SDF-1 $\alpha$ cross-linked collagen group showed more marked binding ability and improved controlled release, compared with the SDF-1 $\alpha$ physically adsorbed collagen group. Co-therapy with SDF- $1 \alpha$ and suboptimal levels of BMP-2 chemically conjugated on collagen scaffolds enhanced ectopic bone formation. This strategy may enable decreases in doses of BMP-2, consequently reducing the risk of side effects and the costs of therapy.

\section{Acknowledgements}

The authors would like to thank all the members of Guangdong Provincial Key Laboratory of Stomatology (Guangdong, China) for their technical assistance. This study was supported by the National Natural Science Foundation of China (grant no. 81100734) and the Natural Science Foundation of Guangdong of China (grant no. S2013010015805).

\section{References}

1. Dimitriou R, Jones E, McGonagle D and Giannoudis PV: Bone regeneration: Current concepts and future directions. BMC Med 9: 66, 2011.

2. Schwartz CE, Martha JF, Kowalski P, Wang DA, Bode R, Li L and Kim DH: Prospective evaluation of chronic pain associated with posterior autologous iliac crest bone graft harvest and its effect on postoperative outcome. Health Qual Life Outcomes 7: 49, 2009.

3. Kim DH, Rhim R, Li L, Martha J, Swaim BH, Banco RJ, Jenis LG and Tromanhauser SG: Prospective study of iliac crest bone graft harvest site pain and morbidity. Spine J 9: 886-892, 2009.

4. Vo TN, Kasper FK and Mikos AG: Strategies for controlled delivery of growth factors and cells for bone regeneration. Adv Drug Deliv Rev 64: 1292-1309, 2012.

5. Schindeler A, McDonald MM, Bokko P and Little DG: Bone remodeling during fracture repair: The cellular picture. Semin Cell Dev Biol 19: 459-466, 2008.

6. Barnes GL, Kostenuik PJ, Gerstenfeld LC and Einhorn TA: Growth factor regulation of fracture repair. J Bone Miner Res 14: $1805-1815,1999$

7. Nauth A, Ristevski B, Li R and Schemitsch EH: Growth factors and bone regeneration: How much bone can we expect? Injury 42 : $574-579,2011$

8. Giannoudis PV and Einhorn TA: Bone morphogenetic proteins in musculoskeletal medicine. Injury 40 (Suppl 3): S1-S3, 2009.

9. Lieberman JR, Daluiski A and Einhorn TA: The role of growth factors in the repair of bone. Biology and clinical applications. J Bone Joint Surg Am 84-A: 1032-1044, 2002.

10. Seeherman H and Wozney JM: Delivery of bone morphogenetic proteins for orthopedic tissue regeneration. Cytokine Growth Factor Rev 16: 329-345, 2005.

11. Argintar E, Edwards S and Delahay J: Bone morphogenetic proteins in orthopaedic trauma surgery. Injury 42: 730-734, 2011.
12. Kroese-Deutman HC, Ruhé PQ, Spauwen PH and Jansen JA: Bone inductive properties of rhBMP-2 loaded porous calcium phosphate cement implants inserted at an ectopic site in rabbits. Biomaterials 26: 1131-1138, 2005.

13. Ishibe T, Goto T, Kodama T, Miyazaki T, Kobayashi S and Takahashi T: Bone formation on apatite-coated titanium with incorporated BMP-2/heparin in vivo. Oral Surg Oral Med Oral Pathol Oral Radiol Endod 108: 867-875, 2009.

14. Yang HS, La WG, Bhang SH, Lee TJ, Lee M and Kim BS: Apatite-coated collagen scaffold for bone morphogenetic protein-2 delivery. Tissue Eng Part A 17: 1-12, 2011.

15. Fu TS, Chang YH, Wong CB, Wang IC, Tsai TT, Lai PL, Chen LH and Chen WJ: Mesenchymal stem cells expressing baculovirus-engineered BMP-2 and VEGF enhance posterolateral spine fusion in a rabbit model. Spine J 15: 2036-2044, 2015.

16. Huang RL, Chen G, Wang W, Herller T, Xie Y, Gu B and Li Q: Synergy between IL- 6 and soluble IL- 6 receptor enhances bone morphogenetic protein-2/absorbable collagen sponge-induced bone regeneration via regulation of BMPRIA distribution and degradation. Biomaterials 67: 308-322, 2015.

17. Hwang HD, Lee JT, Koh JT, Jung HM, Lee HJ and Kwon TG: Sequential treatment with SDF-1 and BMP-2 potentiates bone formation in calvarial defects. Tissue Eng Part A 21: 2125-2135, 2015.

18. Zlotnik A and Yoshie O: Chemokines: A new classification system and their role in immunity. Immunity 12: 121-127, 2000.

19. Bleul CC, Farzan M, Choe H, Parolin C, Clark-Lewis I, Sodroski J and Springer TA: The lymphocyte chemoattractant SDF-1 is a ligand for LESTR/fusin and blocks HIV-1 entry. Nature 382: 829-833, 1996.

20. Feng Y, Broder CC, Kennedy PE and Berger EA: HIV-1 entry cofactor: Functional cDNA cloning of a seven-transmembrane, $\mathrm{G}$ protein-coupled receptor. Science 272: 872-877, 1996.

21. Iannone M, Ventre M, Pagano G, Giannoni P, Quarto R and Netti PA: Defining an optimal stromal derived factor-1 presentation for effective recruitment of mesenchymal stem cells in 3D. Biotechnol Bioeng 111: 2303-2316, 2014.

22. Naderi-Meshkin H, Bahrami AR, Bidkhori HR, Mirahmadi M and Ahmadiankia N: Strategies to improve homing of mesenchymal stem cells for greater efficacy in stem cell therapy. Cell Biol Int 39: 23-34, 2015.

23. Granero-Moltó F, Weis JA, Miga MI, Landis B, Myers TJ, O'Rear L, Longobardi L, Jansen ED, Mortlock DP and Spagnoli A: Regenerative effects of transplanted mesenchymal stem cells in fracture healing. Stem Cells 27: 1887-1898, 2009.

24. Otsuru S, Tamai K, Yamazaki T, Yoshikawa H and Kaneda Y: Circulating bone marrow-derived osteoblast progenitor cells are recruited to the bone-forming site by the CXCR4/stromal cell-derived factor-1 pathway. Stem Cells 26: 223-234, 2008.

25. Hosogane N, Huang Z, Rawlins BA, Liu X, Boachie-Adjei O, Boskey AL and Zhu W: Stromal derived factor-1 regulates bone morphogenetic protein 2-induced osteogenic differentiation of primary mesenchymal stem cells. Int J Biochem Cell Biol 42: 1132-1141, 2010.

26. Zhu W, Boachie-Adjei O, Rawlins BA, Frenkel B, Boskey AL, Ivashkiv LB and Blobel CP: A novel regulatory role for stromal-derived factor-1 signaling in bone morphogenic protein-2 osteogenic differentiation of mesenchymal C2C12 cells. J Biol Chem 282: 18676-18685, 2007.

27. Herberg S, Susin C, Pelaez M, Howie RN, Moreno de Freitas R, Lee J, Cray JJ Jr, Johnson MH, Elsalanty ME, Hamrick MW, et al: Low-dose bone morphogenetic protein-2/stromal cell-derived factor-1 $\beta$ cotherapy induces bone regeneration in critical-size rat calvarial defects. Tissue Eng Part A 20: 1444-1453, 2014.

28. Higashino K, Viggeswarapu M, Bargouti M, Liu H, Titus L and Boden SD: Stromal cell-derived factor-1 potentiates bone morphogenetic protein-2 induced bone formation. Tissue Eng Part A 17: 523-530, 2011.

29. Ratanavaraporn J, Furuya H, Kohara H and Tabata Y: Synergistic effects of the dual release of stromal cell-derived factor- 1 and bone morphogenetic protein-2 from hydrogels on bone regeneration. Biomaterials 32: 2797-2811, 2011.

30. Zhang Q, He QF, Zhang TH, Yu XL, Liu Q and Deng FL: Improvement in the delivery system of bone morphogenetic protein-2: A new approach to promote bone formation. Biomed Mater 7: 45002, 2012. 
31. He Q, Zhao Y, Chen B, Xiao Z, Zhang J, Chen L, Chen W, Deng $F$ and Dai J: Improved cellularization and angiogenesis using collagen scaffolds chemically conjugated with vascular endothelial growth factor. Acta Biomater 7: 1084-1093, 2011.

32. Chen L, He Z, Chen B, Zhao Y, Sun W, Xiao Z, Zhang J, Yang M Gao Z and Dai J: Direct chemical cross-linking of platelet-derived growth factor-BB to the demineralized bone matrix improves cellularization and vascularization. Biomacromolecules 10 3193-3198, 2009.

33. Chen B, Lin H, Wang J, Zhao Y, Wang B, Zhao W, Sun W and Dai J: Homogeneous osteogenesis and bone regeneration by demineralized bone matrix loading with collagen-targeting bone morphogenetic protein-2. Biomaterials 28: 1027-1035, 2007.

34. Kolácná L, Bakesová J, Varga F, Kostáková E, Plánka L, Necas A, Lukás D, Amler E and Pelouch V: Biochemical and biophysical aspects of collagen nanostructure in the extracellular matrix Physiol Res 56 (Suppl 1): S51-S60, 2007.

35. Venugopal $\mathbf{J}$ and Ramakrishna S: Biocompatible nanofiber matrices for the engineering of a dermal substitute for skin regeneration. Tissue Eng 11: 847-854, 2005.

36. Li X, Feng Q, Liu X, Dong W and Cui F: Collagen-based implants reinforced by chitin fibres in a goat shank bone defect model. Biomaterials 27: 1917-1923, 2006.

37. Boccafoschi F, Habermehl J, Vesentini S and Mantovani D: Biological performances of collagen-based scaffolds for vascular tissue engineering. Biomaterials 26: 7410-7417, 2005.

38. Park SN, Kim JK and Suh H: Evaluation of antibiotic-loaded collagen-hyaluronic acid matrix as a skin substitute. Biomaterials 25: 3689-3698, 2004.

39. Jue R, Lambert JM, Pierce LR and Traut RR: Addition of sulfhydryl groups to Escherichia coli ribosomes by protein modification with 2-iminothiolane (methyl 4-mercaptobutyrimidate). Biochemistry 17: 5399-5406, 1978.
40. Geiger M, Li RH and Friess W: Collagen sponges for bone regeneration with rhBMP-2. Adv Drug Deliv Rev 55: 1613-1629, 2003.

41. McQuibban GA, Butler GS, Gong JH, Bendall L, Power C, Clark-Lewis I and Overall CM: Matrix metalloproteinase activity inactivates the CXC chemokine stromal cell-derived factor-1. J Biol Chem 276: 43503-43508, 2001.

42. Gomes ME, Sikavitsas VI, Behravesh E, Reis RL and Mikos AG Effect of flow perfusion on the osteogenic differentiation of bone marrow stromal cells cultured on starch-based three-dimensional scaffolds. J Biomed Mater Res A 67: 87-95, 2003.

43. Richardson TP, Peters MC, Ennett AB and Mooney DJ: Polymeric system for dual growth factor delivery. Nat Biotechnol 19: 1029-1034, 2001.

44. Kanczler JM, Ginty PJ, White L, Clarke NM, Howdle SM, Shakesheff KM and Oreffo RO: The effect of the delivery of vascular endothelial growth factor and bone morphogenic protein-2 to osteoprogenitor cell populations on bone formation. Biomaterials 31: 1242-1250, 2010.

45. Chen FM, Chen R, Wang XJ, Sun HH and Wu ZF: In vitro cellular responses to scaffolds containing two microencapulated growth factors. Biomaterials 30: 5215-5224, 2009.

46. Patel ZS, Young S, Tabata Y, Jansen JA, Wong ME and Mikos AG: Dual delivery of an angiogenic and an osteogenic growth factor for bone regeneration in a critical size defect model. Bone 43 : 931-940, 2008.

47. Simmons CA, Alsberg E, Hsiong S, Kim WJ and Mooney DJ: Dual growth factor delivery and controlled scaffold degradation enhance in vivo bone formation by transplanted bone marrow stromal cells. Bone 35: 562-569, 2004.

48. Herberg S, Kondrikova G, Periyasamy-Thandavan S, Howie RN, Elsalanty ME, Weiss L, Campbell P, Hill WD and Cray JJ: Inkjet-based biopatterning of SDF-1 $\beta$ augments BMP-2-induced repair of critical size calvarial bone defects in mice. Bone 67: 95-103, 2014 